\title{
Defeasible reasoning in Description Logics: an overview on $\mathcal{D} \mathcal{L}^{\mathrm{N}}$
}

\author{
Piero A. Bonatti ${ }^{1}$, Iliana M. Petrova ${ }^{1}$, and Luigi Sauro ${ }^{1}$ \\ Dep. of Electrical Engineering and Information Technologies, Università di Napoli \\ Federico II, Italy
}

\begin{abstract}
D} \mathcal{L}^{\mathrm{N}}$ is a recent approach that extends description logics with defeasible reasoning capabilities. In this paper we provide an overview on $\mathcal{D} \mathcal{L}^{\mathrm{N}}$, illustrating the underlying knowledge engineering requirements as well as the characteristic features that preserve $\mathcal{D} \mathcal{L}^{\mathrm{N}}$ from some recurrent semantic and computational drawbacks. We also compare $\mathcal{D} \mathcal{L}^{\mathrm{N}}$ with some alternative nonmonotonic semantics, enlightening the relationships between the KLM postulates and $\mathcal{D} \mathcal{L}^{\mathrm{N}}$.
\end{abstract}

\section{Introduction}

In complex areas such as law and science, knowledge has been in centuries formulated by primarily describing prototypical instances and properties, and then by overriding the general theory to include possible exceptions. For example, many laws are formulated by adding new norms that, in case of conflicts, may partially or completely override the previous ones. Similarly, biologists have been incrementally introducing exceptions to general properties. For instance, the human heart is usually located in the left-hand half of the thorax. Still there are exceptional individuals, with so-called situs inversus, whose heart is located on the opposite side. Eukariotic cells are those with a proper nucleus, by definition. Still they comprise mammalian red blood cells, that in their mature stage have no nucleus 1

Also many modern applications and methodologies in Computer Science rely on some sort of overriding mechanism. In Object Oriented Programming the definitions in a subclass may override any conflicting bindings belonging to its superclasses. Analogously, formal languages designed to describe role-based access control or other privacy policies generally allow to formulate default conditions, such as open and closed policies 2 conflict resolution methods such as denials take precedence, and authorization inheritance with exceptions [14.

Summarizing, the mentioned fields manifest to a large extent different forms of defeasible knowledge where general axioms can be recanted in special cases by employing some suitable overriding mechanism. Nevertheless, this natural approach cannot be directly adopted in designing Semantic Web ontologies. In

\footnotetext{
${ }^{1}$ All of these examples are introduced and discussed in 3941.

2 If no explicit authorization has been specified for a given access request, then an open policy permits the access while a closed policy denies it.
} 
fact, the underlying descriptions logics (DLs), which are based on the monotonic semantics of FOL, do not allow to express and reason on defeasible knowledge and exceptions. Consequently, several authors advocated nonmonotonic logics as a useful means to address this limitation and proposed different formalisms based on circumscription [10/9/11, autoepistemic logic 24/25], typicality operators 2628131, or rational closure 292016, just to mention a few.

In this context, $\mathcal{D} \mathcal{L}^{\mathrm{N}} 711513$ is a recent family of nonmonotonic DL specifically designed to meet the knowledge engineering requirements that come from the aforesaid application domains. $\mathcal{D} \mathcal{L}^{\mathrm{N}}$ is prototype oriented: it uses $\mathrm{N} C$ to denote the normal/prototypical instances of a concept $C$, and extends terminological axioms with prioritized defeasible inclusions (DIs) $C \sqsubseteq_{n} D$.

A difficulty arising at a design level is that the notion itself of prototype can be inherently ambiguous. Prototypes may represent in a frequentistic fashion the properties that are shared by the majority of the instances, or they can differently be interpreted idealistically as platonic models that might not exist in the real world due to their degree of perfection. $\mathcal{D} \mathcal{L}^{\mathrm{N}}$ does not aim at encompassing all different, and philosophically interesting, notions of prototype; being plainly application-oriented, it is rather inspired by what McCarthy calls communication and database storage conventions [37. In this perspective, a prototype $\mathrm{NC}$ is meant to factorize the common features of the concept $C$ and confine exceptional subclasses to an explicit detailed axiomatization (so as to reduce the size and cost of knowledge bases and improve their readability). Thus, defeasible inclusions $C \sqsubseteq_{n} D$ mean (roughly speaking): "by default, all prototypical instances that satisfy $C$ satisfy also D, unless stated otherwise", that is, unless some higher priority axioms contradict this implication. If such a contradiction arises, then $C \sqsubseteq_{n} D$ is overridden. The standard/prototypical instances of $C$ are required to satisfy all the DIs that are not overridden in $C$.

As mentioned above, also other nonmonotonic logics support defeasible inheritance with overriding in general. Nevertheless, in each of these previous approaches, either some desiderable features are missing or some natural inferences do not hold. Moreover, they are generally based on complex semantics which make defeasible reasoning difficult to track 3 In this respect, DLN's behavior is easier to grasp, and is expected to facilitate knowledge engineers in formulating and validate ontologies at a large scale, while producing the expected conclusions.

Finally, apart from rational closure and restricted forms of typicality [30, defeasible reasoning significantly increases the computational complexity of standard reasoning tasks even in low-complexity description logics 27/812. Conversely, in all DL fragments of pratical interest, $\mathcal{D} \mathcal{L}^{\mathrm{N}}$ does not manifest a higher complexity with respect to the classical counterpart. Moreover, efficiency can be further enhanced through a range of optimization techniques, including modularization [13].

\footnotetext{
${ }^{3}$ For example, circumscription identifies, in case of conflicting nonmonotonic axioms, all optimal repairs and then computes the inferences that hold for all repairs.
} 


\begin{tabular}{|l|c|l|}
\hline Name & Syntax & Semantics \\
\hline \hline top & $\top$ & $\Delta^{\mathcal{I}}$ \\
\hline bottom & $\perp$ & $\emptyset$ \\
\hline negation & $\neg C$ & $\Delta^{\mathcal{I}} \backslash C^{\mathcal{I}}$ \\
\hline conjunction & $C \sqcap D$ & $C^{\mathcal{I}} \cap D^{\mathcal{I}}$ \\
\hline disjunction & $C \sqcup D$ & $C^{\mathcal{I}} \cup D^{\mathcal{I}}$ \\
\hline$\exists$ restriction & $\exists R . C$ & $\left\{d \in \Delta^{\mathcal{I}} \mid \exists e \in \Delta^{\mathcal{I}} \cdot\left[(d, e) \in R^{\mathcal{I}} \wedge e \in \mathcal{C}^{\mathcal{I}}\right]\right\}$ \\
\hline$\forall$ restriction & $\forall R . C$ & $\left\{d \in \Delta^{\mathcal{I}} \mid \forall e \in \Delta^{\mathcal{I}} \cdot\left[(d, e) \in R^{\mathcal{I}} \rightarrow e \in \mathcal{C}^{\mathcal{I}}\right]\right\}$ \\
\hline
\end{tabular}

Fig. 1. Syntax and semantics of some common constructs.

This paper is meant to illustrate $\mathcal{D} \mathcal{L}^{\mathrm{N}}$ and its main features, extending previous discussions of $\mathcal{D} \mathcal{L}^{\mathrm{N}}$, s properties with some recent contributions to rational closure. The paper is organized as follows. In the next section we briefly recall the basics of monotonic description logics. In Section 3 we introduce $\mathcal{D} \mathcal{L}^{\mathrm{N}}$ and provide a few examples of knowledge bases and inference. Section 4 compares $\mathcal{D} \mathcal{L}^{\mathrm{N}}$ with the other major nonmonotonic DLs in terms of practical engineering requirements, and in terms of logical properties, centred around the KLM postulates. The paper is concluded by a summary and a list of interesting topics for further work.

\section{Preliminaries}

Description logics are a family of formal languages representing the logical foundations of the W3C Ontology Web Language (OWL2). They offer a variegated set of logical constructors and axioms that balance between expressiveness and computational complexity according to the application needs. Due to space limitations, we refer to 5 for a comprehensive overview. Here, we just introduce the DL fragment $\mathcal{A L C}$, which allows to understand the examples that will follow.

An alphabet or signature consists of a set $\mathrm{N}_{C}$ of concept names, a set $\mathrm{N}_{\mathrm{R}}$ of role names, and (possibly) a set $\mathrm{N}_{1}$ of individual names (all countably infinite). Thereafter, metavariables $A, B$ will range over concept names, $R$ and $S$ over roles, and $a, b$ and $d$ over individual names. The term predicate will refer to a generic element of $N_{C} \cup N_{R}$.

In DLs, a wide range of operators allow to inductively formulate compound concepts. The logic $\mathcal{A L C}$, in particular, compound concepts are defined by the following grammar:

$$
C, D::=A|\top| \perp|\neg C| C \sqcap D|C \sqcup D| \exists R . C \mid \forall R . C .
$$

Note, however, that our framework applies also to more expressive DLs such as $\mathcal{S R O I} \mathcal{I}(\mathcal{D})$ that constitutes the foundation of the full standard OWL2.

The semantics of DLs is defined in terms of interpretations $\mathcal{I}=\left\langle\Delta^{\mathcal{I}},{ }^{\mathcal{I}}\right\rangle$. The domain $\Delta^{\mathcal{I}}$ is a non-empty set of individuals and the interpretation function. ${ }^{\mathcal{I}}$ 
maps each concept name $A \in \mathrm{N}_{\mathrm{C}}$ to a subset $A^{\mathcal{I}}$ of $\Delta^{\mathcal{I}}$, each role name $R \in \mathrm{N}_{\mathrm{R}}$ to a binary relation $R^{\mathcal{I}}$ on $\Delta^{\mathcal{I}}$, and each individual name $a \in \mathrm{N}_{\text {I }}$ to an individual $a^{\mathcal{I}} \in \Delta^{\mathcal{I}}$. The extension of ${ }^{\mathcal{I}}$ to $\mathcal{A L C}$ compound concepts is inductively defined as shown in the third column of Figure 1, An interpretation $\mathcal{I}$ is called a model of a concept $C$ if $C^{\mathcal{I}} \neq \emptyset$.

A (general) TBox is a finite set of concept inclusions (CIs) $C \sqsubseteq D$. As usual, we use $C \equiv D$ as an abbreviation for $C \sqsubseteq D$ and $D \sqsubseteq C$. An $A B o x$ is a finite set of concept assertions $C(a)$ and role assertions $R(a, b)$. An interpretation $\mathcal{I}$ satisfies (i) a CI $C \sqsubseteq D$ if $C^{\mathcal{I}} \subseteq D^{\mathcal{I}}$, (ii) an assertion $C(a)$ if $a^{\mathcal{I}} \in C^{\mathcal{I}}$, and (iii) an assertion $R(a, b)$ if $\left(a^{\mathcal{I}}, b^{\mathcal{I}}\right) \in R^{\mathcal{I}}$. Then, $\mathcal{I}$ is a (classical) model of a TBox $\mathcal{T}$ (resp. an ABox $\mathcal{A}$ ) if $\mathcal{I}$ satisfies all the members of $\mathcal{T}$ (resp. $\mathcal{A}$ ).

In this paper, we will sometimes mention some important DLs that have been extensively studied in the literature and constitute the foundation of semantic web standards. The logic $\mathcal{E} \mathcal{L}$ supports only $\top, \sqcap$, and $\exists$. Its extension $\mathcal{E} \mathcal{L}^{\perp}$ supports also $\perp$. The logic $\mathcal{E} \mathcal{L}^{++}$further adds concrete domains and some expressive role inclusions (see [2] for further details).

The logic DL-lite $_{R}$ [17] supports inclusions shaped like $C \sqsubseteq D$ and $C \sqsubseteq \neg D$, where $C$ and $D$ range over concept names and unqualified existential restrictions such as $\exists R$ and $\exists R^{-}$(where $R^{-}$is the inverse of role $R$ ). $\mathcal{E} \mathcal{L}^{++}$and DL-lite $_{R}$, respectively, constitute the foundation of the OWL2 profiles OWL2EL and OWL2-QL. Both play an important role in applications; their inference problems are tractable (the same holds for some extensions of DL-lite ${ }_{R}$, see [1]).

Finally, we will use in Section 4.2 boolean combinations of assertions and inclusions. Although these axioms are not directly allowed in DLs, they can be simulated in $\mathcal{S R O I} \mathcal{Q}(\mathcal{D})$ through the universal role $U$. For example, $\neg(C \sqsubseteq D)$ and $\left(C_{1} \sqsubseteq D_{1}\right) \vee\left(C_{2} \sqsubseteq D_{2}\right)$ can be expressed as $\top \sqsubseteq \exists U .(C \sqcap \neg D)$ and $\top \sqsubseteq$ $\left(\forall U .\left(\neg C_{1} \sqcup D_{1}\right)\right) \sqcup\left(\forall U .\left(\neg C_{2} \sqcup D_{2}\right)\right)$, respectively.

\section{$3 \quad$ The defeasible logic $\mathcal{D} \mathcal{L}^{\mathrm{N}}$}

Given a classical description logic language $\mathcal{D} \mathcal{L}$, let $\mathcal{D} \mathcal{L}^{\mathrm{N}}$ be the extension of $\mathcal{D} \mathcal{L}$ with a new concept name $\mathrm{N} C$ for each $\mathcal{D} \mathcal{L}$ concept $C$. $\mathrm{N} C$ is called a normality concept and denotes the normal instances of $C$.

A $\mathcal{D} \mathcal{L}^{\mathrm{N}}$ knowledge base is a disjoint union $\mathcal{K B}=\mathcal{S} \cup \mathcal{D}$ such that

- $\mathcal{S}$ is a finite set of $\mathcal{D} \mathcal{L}$ concept inclusions and assertions;

$-\mathcal{D}$ is a finite set of defeasible inclusions (DIs, for short) $C \sqsubseteq_{n} D$ where $C$ and $D$ are $\mathcal{D} \mathcal{L}^{\mathrm{N}}$ concepts.

Thereafter, given a DI $\delta=C \sqsubseteq_{n} D$, by $\operatorname{pre}(\delta)$ and $\operatorname{con}(\delta)$ we denote $C$ and $D$, respectively. A knowledge base is canonical if $\operatorname{pre}(\delta)$ does not contain normality concepts, for all $\delta \in \mathcal{D}$.

Roughly, $C \sqsubseteq_{n} D$ means: "the normal instances of $C$ are instances of $D$, unless stated otherwise by some higher priority axioms". As mentioned in the introduction DIs have an utilitarian purpose. They are meant to factorize the 
common properties that hold for normal entities, so as to minimize the amount of knowledge that must be explicitly encoded.

Defeasible inclusions are prioritized by a strict partial order $\prec$ over $\mathcal{D}$. The intended meaning of $\delta_{1} \prec \delta_{2}$ is that $\delta_{1}$ has higher priority than $\delta_{2}$ and, in case of conflicts, it is preferable to sacrifice $\delta_{2} . \mathcal{D} \mathcal{L}^{\mathrm{N}}$ solves automatically only the conflicts that can be settled using $\prec$. Any other conflict shall be resolved by the knowledge engineer (typically by adding specific DIs). Here, we focus on a priority relation which is determined by so-called specificity. Roughly speaking, specificity states that, in case of conflicts, the specific properties of $\operatorname{pre}\left(\delta_{1}\right)$ override those of the more general concept $\operatorname{pre}\left(\delta_{2}\right)$ :

$$
\delta_{1} \prec \delta_{2} \text { iff } \mathcal{S} \models \operatorname{pre}\left(\delta_{1}\right) \sqsubseteq \operatorname{pre}\left(\delta_{2}\right) \text { and } \mathcal{S} \not \models \operatorname{pre}\left(\delta_{2}\right) \sqsubseteq \operatorname{pre}\left(\delta_{1}\right) .
$$

Note that $\mathcal{D} \mathcal{L}^{\mathrm{N}}$ is largely parametric with respect to which priority relation is used. An alternative choice could be, for example, a priority relation based on the ranking function of rational closure adopted in [20].

Due to space limitations, we refer to 7 for the model-theoretic semantics of $\mathcal{D} \mathcal{L}^{\mathrm{N}}$. Here, we present only its reduction to classical reasoning.

Let $\mathcal{K} \mathcal{B}=\mathcal{S} \cup \mathcal{D}$ be a $\mathcal{D} \mathcal{L}^{\mathrm{N}}$ knowledge base and $\alpha$ a query of interest, that can be either a $\mathrm{CI}$ or an assertion. By $\mathcal{K B} \approx \alpha$ we mean that $\alpha$ is a $\mathcal{D} \mathcal{L}^{\mathrm{N}}$ semantic consequence of $\mathcal{K B}$.

The classical reduction of $\approx$ requires some preliminary notions:

- For all DIs $\delta \in \mathcal{D}$ and all normality concepts $\mathrm{N} C \in \Sigma$, let

$$
\delta^{\mathrm{NC}}=(\mathrm{N} C \sqcap \operatorname{pre}(\delta) \sqsubseteq \operatorname{con}(\delta)) ;
$$

- for all sets of $\mathcal{D} \mathcal{L}$ axioms $\mathcal{S}^{\prime}$ and all DIs $\delta$, let $\mathcal{S}^{\prime} \downarrow \prec \delta$ denote the result of removing from $\mathcal{S}^{\prime}$ all the axioms $\delta_{0}^{\mathrm{NC}}$ such that $\delta_{0}$ 's priority is not higher than $\delta$ 's:

$$
\mathcal{S}^{\prime} \downarrow \prec \delta=\mathcal{S}^{\prime} \backslash\left\{\delta_{0}^{\mathrm{N} C} \mid \mathrm{N} C \in \Sigma \wedge \delta_{0} \nprec \delta\right\} ;
$$

- finally, let $\delta_{1}, \ldots, \delta_{|\mathcal{D}|}$ be an arbitrary linearization of $(\mathcal{D}, \prec)$, which means that $\left\{\delta_{1}, \ldots, \delta_{|\mathcal{D}|}\right\}=\mathcal{D}$ and for all $i, j=1, \ldots,|\mathcal{D}|$, if $\delta_{i} \prec \delta_{j}$ then $i<j$.

Then, $\mathcal{K B} \approx \alpha$ holds iff $\mathcal{K B}^{\Sigma} \models \alpha$, where $\Sigma$ is the set of normality concepts occurring in both $\mathcal{K B}$ and $\alpha$, and $\mathcal{K} \mathcal{B}^{\Sigma}$ is the classical knowledge base resulting from the following inductive construction (where $i=1,2, \ldots,|\mathcal{D}|$ ):

$$
\begin{aligned}
\mathcal{S}_{0}^{\Sigma} & =\mathcal{S} \cup\{\mathrm{N} C \sqsubseteq C \mid \mathrm{N} C \in \Sigma\} \\
\mathcal{S}_{i}^{\Sigma} & =\mathcal{S}_{i-1}^{\Sigma} \cup\left\{\delta_{i}^{\mathrm{N} C} \mid \mathrm{N} C \in \Sigma \text { and } \mathcal{S}_{i-1}^{\Sigma} \downarrow \prec \delta_{i} \cup\left\{\delta_{i}^{\mathrm{N} C}\right\} \not \mid \mathrm{N} C \sqsubseteq \perp\right\} \\
\mathcal{K B}^{\Sigma} & =\mathcal{S}_{|\mathcal{D}|}^{\Sigma} .
\end{aligned}
$$

In informal terms, the first step extends $\mathcal{S}$ with the axioms $\mathrm{N} C \sqsubseteq C$ stating that the normal instances of $C$ are a fortiori instances of $C$. The construction proceeds by processing the DIs $\delta_{i} \in \mathcal{D}$ in decreasing priority order; if adding $\delta_{i}$ to the (higher priority) $\delta_{j} \prec \delta_{i}$ that have been previously selected does not make $\mathrm{NC}$ inconsistent, as stated by (3), then $\delta_{i}^{\mathrm{NC}}$ is included in $\mathcal{K B}^{\Sigma}$, otherwise $\delta_{i}^{\mathrm{NC}}$ is discarded (overridden). 
Example 1. Recall that situs inversus refers to humans whose heart is on the right-hand side of the thorax, differently from typical humans whose heart is on the opposite side. If we stipulate that no heart can be simultaneously located on both sides, then a simple axiomatization is:

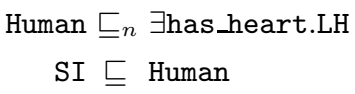

SI $\sqsubseteq$ Human



$\exists$ has_heart.LH $\sqsubseteq \neg \exists$ has_heart.RH

where LH (resp. RH) denotes left-positioned (resp. right-positioned) hearts and SI stands for situs inversus.

Since $\mathcal{S} \subseteq \mathcal{K B}^{\Sigma}$, by (7) and (8) we have that the instances of SI have their heart on the right-hand side (and hence not on the left-hand side):

$$
\mathcal{K B} \approx \mathrm{SI} \sqsubseteq \exists \text { has heart.RH } \quad(9) \quad \mathcal{K B} \approx \mathrm{SI} \sqsubseteq \neg \exists \text { has heart.LH . (10) }
$$

Then, let $\Sigma=\{$ NHuman $\}$. It is straightforward to see that $\mathcal{K B}^{\Sigma}$ consists of the strong axioms (6) - (8), plus

$$
\text { NHuman } \sqsubseteq \text { Human }
$$

Human $\sqcap$ NHuman $\sqsubseteq \exists$ has_heart.LH .(12)

Consequently, we have that

$$
\mathcal{K B} \approx \text { NHuman } \sqsubseteq \exists \text { has_heart.LH . }
$$

Moreover, as a classical consequence of the above inferences, one can further conclude that people with situs inversus are not standard humans:

$$
\mathcal{K B} \approx \mathrm{SI} \sqsubseteq \neg \text { NHuman . }
$$

Conversely, if $\Sigma=\{\mathrm{NSI}\}$, the iterative construction of $\mathcal{K B}^{\Sigma}$ adds in first step the axiom

$$
\mathrm{NSI} \sqsubseteq \mathrm{SI},
$$

then the DI (5) is overridden, since adding

$$
\text { Human } \sqcap \text { NSI } \sqsubseteq \exists \text { has_heart.LH }
$$

would make, together with axioms (6), (7), (8), and (15), NSI inconsistent. Consequently, NSI is simply a consistent subclass of SI that does not satisfy any further property.

Now, extend $\mathcal{K} \mathcal{B}$ with the additional DI:

$$
\text { Human } \sqsubseteq_{n} \exists \text { has_organ.Nose . }
$$

Note that (17) and (5) have both maximal priority (indeed, they are incomparable by specificity). It is easy to see that (17) is overridden neither in NHuman nor in NSI, therefore both of the following inferences are valid:

$$
\begin{aligned}
& \mathcal{K B} \approx \text { NHuman } \sqsubseteq \exists \text { has_organ.Nose } \\
& \mathcal{K B} \approx \text { NSI } \sqsubseteq \text { has_organ.Nose. }
\end{aligned}
$$

In other words, the property of having a nose is inherited even if (14) make SI exceptional w.r.t. Human. 
Example 2. Consider the following variant of Nixon's diamond [7:

$$
\begin{aligned}
& \text { Quaker } \sqsubseteq_{n} \text { Pacifist, } \quad(20) \\
& \text { Republican } \sqsubseteq_{n} \neg \text { Pacifist, (21) } \\
& \text { RepQuaker } \sqsubseteq \text { Republican } \sqcap \text { Quaker . }
\end{aligned}
$$

Note that the two DIs (20) and (21) are incomparable under specificity. Moreover, since they both can be individually satisfied by NRepQuaker, without making it inconsistent, none of them is overridden in NRepQuaker. It follows that NRepQuaker must satisfy both DIs and consequently RepQuaker is associated to an inconsistent prototype:

$$
\mathcal{K B} \approx \text { NRepQuaker } \sqsubseteq \perp
$$

Note that even if the prototype of RepQuaker is inconsistent, the knowledge base is consistent, as well as many normality concepts. In particular, we have $\mathcal{K B} \not \notin$ NQuaker $\sqsubseteq \perp$ and $\mathcal{K B} \not \notin$ NRepublican $\sqsubseteq \perp$.

Moreover, consequence (23) cannot be resolved by logic since (20) and (21) are perfectly symmetric w.r.t. NRepQuaker. Removing the inconsistency is up to the knowledge engineer which has to choose how to repair $\mathcal{K B}$. For instance, adding RepQuaker $\sqsubseteq_{\mathrm{n}}$ Pacifist (resp. RepQuaker $\sqsubseteq_{\mathrm{n}} \neg$ Pacifist) resolves the conflict in favor of the first (resp. second) DI.

Remark 1. The other nonmonotonic semantics of DLs silently "hide" unresolved conflicts, by deriving none of the conflicting properties. The result is a gap in the knowledge base. For example, Nixon was notoriously not a pacifist, and until the conflict is resolved, this information is not accessible to reasoners. In other examples, such knowledge gaps may have important consequences 7 . Unlike the other nonmonotonic logics, $\mathcal{D} \mathcal{L}^{\mathrm{N}}$ helps knowledge engineers in identifying the gaps caused by unresolved conflicts. Searching for inconsistent prototypes is analogous to the classical KB debugging activity consisting in identifying inconsistent concepts, and all engines support it.

Example 3. In several countries (e.g. Mexico, Norway and Brazil) military service is mandatory for male citizens (except for special cases such as mental disorders). After military training, citizens become reservists, and shall join the army again in case of war. This can be formalized with the following DIs:

$$
\begin{gathered}
\text { MaleCitizen } \sqsubseteq_{n} \text { HasMilitaryTraining } \\
\text { MaleCitizen } \sqcap \text { HasMilitaryTraining } \sqsubseteq_{n} \text { Reservist. }
\end{gathered}
$$

The exceptions to the above rules include minors:

$$
\begin{aligned}
& \text { MinorMaleCitizen } \sqsubseteq \text { MaleCitizen } \\
& \text { MinorMaleCitizen } \sqsubseteq \neg \text { HasMilitaryTraining. }
\end{aligned}
$$

Axiom (27) should prevent (25) from being applied to minors, that is, it should not be possible to conclude that NMinorMaleCitizen $\sqsubseteq$ Reservist (indeed, this is what happens with $\mathcal{D} \mathcal{L}^{\mathrm{N}}$ ). 
For what concerns the computational complexity of $\mathcal{D} \mathcal{L}^{\mathrm{N}}$, notice that the iterative construction of $\mathcal{K B}^{\Sigma}$ requires, at each step $i \in\{1, \ldots,|\mathcal{D}|\}$, (i) to restrict $\mathcal{S}_{i}^{\Sigma}$ to the DIs that have a higher priority than $\delta_{i}$, and (ii) to evaluate $|\Sigma|$ consistency checks. If the priority relation is based on specificity, checking whether $\delta_{j} \prec \delta_{i}$ consists in solving two subsumption problems and hence it has the same complexity as entailment in $\mathcal{D} \mathcal{L}$. Also the second point simply comes down to classical reasoning in the underlying $\mathcal{D} \mathcal{L}$. Consequently, $\mathcal{D} \mathcal{L}^{\mathrm{N}}$ entailment has the same complexity as in $\mathcal{D} \mathcal{L}$. In general, considering that different priority relations can be used, the following characterization holds.

Theorem 1. Let $\mathcal{D} \mathcal{L}$ be a $D L$ fragment such that subsumption (resp. instance) checking in $\mathcal{D} \mathcal{L}$ belongs to a complexity class $\mathcal{C}$, and deciding the preference relation $\prec$ belongs to $\mathrm{P}^{\mathcal{C}} 4$ If $\mathcal{D} \mathcal{L}$ supports $\square$ in the left-hand side of inclusions, then subsumption (resp. instance) checking in $\mathcal{D} \mathcal{L}^{\mathrm{N}}$ is in $\mathrm{P}^{\mathcal{C}}$.

Since $\mathrm{P}^{\mathrm{P}}$ equals $\mathrm{P}$, the entailment problem $\mathcal{K} \mathcal{B} \approx \alpha$ is tractable in low complexity description logics such as $\left(D L \text {-lite } \text { horn }^{(\mathcal{H N})}\right)^{\mathrm{N}}\left[1\right.$ and $\left(\mathcal{E} \mathcal{L}^{++}\right)^{\mathrm{N}}$. Similarly, Theorem 1 tells us that $\mathcal{S R O I} \mathcal{Q}^{\mathrm{N}}$ reasoning is in $\mathrm{P}^{\mathrm{N} 2 \operatorname{Exp} T i m e}$ for suitable priority relations.

\section{Features and comparisons}

\subsection{Knowledge engineering requirements}

The $\mathcal{D} \mathcal{L}^{\mathrm{N}}$ family of logics results from a utilitarian way of approaching nonmonotonic logic design. The main goal of this approach is addressing the practical needs of ontology and policy designers, that have been illustrated with several examples in the literature on biomedical ontologies and semantic web policies. Here is a summary of the main shortcomings addressed by $\mathcal{D} \mathcal{L}^{\mathrm{N}}$ (see [7] for more details and explanations):

- Inheritance blocking. Most of the logics grounded on preferential semantics and rational closure block the inheritance of all default properties towards exceptional subclasses (as opposed to overriding only the properties that are modified in those subclasses). $\mathcal{D} \mathcal{L}^{\mathrm{N}}$, s overriding mechanism does not suffer from this drawback (see Example 1).

- Undesired CWA effects. Many nonmonotonic DLs extend default properties to as many individuals as possible, thereby introducing CWA (i.e. closedworld assumption) effects that clash with the intended behavior of ontologies. For instance, exceptional concepts (such as SI in Example 1) collapse to the list of constants that are explicitly asserted to be in the concept (and if no such constants exist, exceptional concepts become inconsistent). $\mathcal{D} \mathcal{L}^{\mathrm{N}}$ does not introduce any CWA effect because it does not force individuals to be normal, unless explicitly stated otherwise.

${ }^{4} \mathrm{P}^{\mathcal{C}}$ is the class of all problems that can be solved by a deterministic Turing machine in polynomial time using an oracle for $\mathcal{C}$. 
- Control on priorities. Since priorities are not fixed a priori in $\mathcal{D} \mathcal{L}^{\mathrm{N}}$, knowledge engineers can adapt them to their needs. In principle, it is possible to override DIs based on temporal criteria (which may be useful in legal ontologies and ontology versioning), define default conflict resolution criteria, and even use rational closure's specificity-based axiom ranking. The logics derived from inheritance networks, preferential semantics, and rational closure can only support their fixed, specificity-based overriding criterion.

- Default role fillers. Should role values be restricted to normal individuals? Sometimes, this kind of inference is desirable, sometimes it is not, cf. 7 . Some logics are completely unable to apply default properties to role values 5 Some others cannot switch this inference off when it is not desired. Only $\mathcal{D} \mathcal{L}^{\mathrm{N}}$ and $\mathcal{A L C}+\mathbf{T}_{\text {min }}$ make it possible to control this kind of inference. Due to its explicit priorities, $\mathcal{D} \mathcal{L}^{\mathrm{N}}$ is also able to encode a design pattern that makes role ranges normal whenever this does not override any explicit DI.

- Inconsistent prototype detection. We argued that when conflicts cannot be settled by priorities, silent conflict resolution is not a desirable feature: knowledge engineers should be involved because there is no universally correct automated resolution criterion (cf. Example 2). Only $\mathcal{D} \mathcal{L}^{\mathrm{N}}$ and probabilistic description logics (and $\mathcal{A L C}+\mathbf{T}_{\min }$, in some very specific cases) detect inconsistent prototypes and make them evident, as advocated in Remark 1.

- Unique deductive closure. As a result of automated conflict resolution, several nonmonotonic logics yield multiple deductive closures, corresponding to all the alternative ways of solving each conflict. $\mathcal{D} \mathcal{L}^{\mathrm{N}}$ is one of the logics that has a unique closure.

- Generality. Nonmonotonic extensions should be applicable to all description logics, or at least to the standard OWL2-DL (i.e. the logic $\mathcal{S} \mathcal{R O I} \mathcal{Q}(\mathcal{D})$ ). Typicality logics and rational closure, instead, are limited to logics that satisfy the disjoint union model property. Recently, it has been shown that for expressive DLs that do not enjoy this property, syntactic inference does not match semantics [6. The same paper introduces stable rational closure that solves the generality problem for rational closure, but re-introduces the issue of multiple (or non existent) deductive closures. It is currently not clear how to design a logic that satisfies the KLM postulates, is fully general, and yields a unique closure for all knowledge bases.

- Low complexity. $\mathcal{D} \mathcal{L}^{\mathrm{N}}$ preserves the tractability of these reasoning tasks for all low-complexity DLs, including the rich tractable logics $\mathcal{E L}^{++}$and DL$\operatorname{lite}_{\text {Horn }}^{(\mathcal{H N})}$. Currently, no other nonmonotonic DL enjoys this property to the same extent. Rational closure has been proved to be tractable for $\mathcal{E} \mathcal{L}$ extended with $\perp 2338$. Some logics, such as 20/1922/31/28, preserve the asymptotic complexity of ExpTime-complete DLs like $\mathcal{A L C}$. More generally, $\mathcal{D} \mathcal{L}^{\mathrm{N}}$ preserves the asymptotic complexity of all the DLs that belong to a

\footnotetext{
5 This is the case for rational closure. Recently, in 38, a solution has been proposed for $\mathcal{E} \mathcal{L}$ with $\perp$. It is unclear how to extend it to more expressive DLs, and it is not possible to "turn off" the application of default rules to role fillers.
} 
deterministic complexity class that contains P. For nondeterministic complexity classes $\mathcal{C}$, an upper bound is $P^{\mathcal{C}}$.

$\mathcal{D} \mathcal{L}^{\mathrm{N}}$ has been designed to address the above practical issues. In general, the utilitarian approach led us to make $\mathcal{D} \mathcal{L}^{\mathrm{N}}$ neutral with respect to the inferences that are not always desired: when possible, $\mathcal{D} \mathcal{L}^{\mathrm{N}}$ gives knowledge engineers the ability of switching those inferences on and off. The final result of this investigation is a logic that enjoys a unique set of properties, as shown by the summary in Table 1.

Table 1. Summary of comparisons with nonmonotonic DLs

\begin{tabular}{|c|c|c|c|c|c|c|c|c|}
\hline Features & $\begin{array}{l}\text { CIRC } \\
1211 \\
\end{array}$ & $\begin{array}{r}\mathrm{DEF} \\
34 \\
\end{array}$ & \begin{tabular}{|c|} 
AEL \\
25 \\
\end{tabular} & $\begin{array}{r}\text { TYP } \\
3128 \\
\end{array}$ & 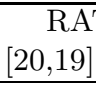 & 22 & $\begin{array}{r}\mathrm{PR} \\
36 \\
\end{array}$ & $\mathcal{D} \mathcal{L}^{\mathrm{N}}$ \\
\hline $\begin{array}{l}\text { no inheritance } \\
\text { blocking }\end{array}$ & $\checkmark$ & $\checkmark$ & $\checkmark$ & & & $\checkmark$ & $\checkmark$ & $\checkmark$ \\
\hline no CWA effects & & $\sqrt{ }$ & $\sqrt{ }$ & & $\bar{\checkmark}$ & $\checkmark$ & & $\bar{\checkmark}$ \\
\hline $\begin{array}{l}\text { fine-grained } \\
\text { control } \\
\text { on role ranges }\end{array}$ & & & & $\checkmark^{(1)}$ & & & & $\checkmark$ \\
\hline $\begin{array}{ll}\text { detects } & \text { in- } \\
\text { consistent } & \\
\text { prototypes } & \end{array}$ & & & & $\checkmark^{(1)}$ & & & $\checkmark^{(2)}$ & $\checkmark$ \\
\hline $\begin{array}{l}\text { unique deductive } \\
\text { closure }\end{array}$ & $\checkmark$ & & & $\checkmark$ & & & & $\checkmark$ \\
\hline $\begin{array}{l}\text { preserves tractabil- } \\
\text { ity }\end{array}$ & & & & & & & & $\checkmark$ \\
\hline generality & $\checkmark$ & $\checkmark$ & $\checkmark$ & & & & $\checkmark$ & $\checkmark$ \\
\hline implicit specificity & & & & $\bar{\checkmark}$ & $\bar{\checkmark}$ & $\checkmark$ & $\bar{\checkmark}$ & \\
\hline other priorities & $\checkmark$ & $\sqrt{ }$ & & & & & & $\checkmark$ \\
\hline
\end{tabular}

(1) Partially supported.

(2) Inconsistency may propagate to the entire KB.

We deliberately refrained from adding a priori any requirements that are not directly motivated by applications, such as the KLM postulates. Interestingly, $\mathcal{D} \mathcal{L}^{\mathrm{N}}$ satisfies many of those postulates, though, as illustrated in the next section.

\section{$4.2 \mathcal{D} \mathcal{L}^{\mathrm{N}}$ and the KLM axioms}

In this section we analyze the logical properties of $\mathcal{D} \mathcal{L}^{\mathrm{N}}$ through the KLM postulates. In [33 35134, Kraus, Lehmann, and Magidor argued that in order to reason about what normally holds in the world, it is desirable to make nonmonotonic consequence relations closed under certain properties, called KLM postulates. 
Table 2. The KLM postulates in $\mathcal{D} \mathcal{L}^{\mathrm{N}}$

\begin{tabular}{|c|c|c|}
\hline Name & Rule schema & Sound in $\mathcal{D} \mathcal{L}^{\mathrm{N}}$ \\
\hline REF & $\frac{\alpha \in \mathcal{K} \mathcal{B}}{\mathcal{K} \mathcal{B} \approx \alpha}$ & $\checkmark$ \\
\hline $\mathrm{CT}$ & $\frac{\mathcal{K} \mathcal{B} \approx \alpha \quad \mathcal{K B} \cup\{\alpha\} \approx \gamma}{\mathcal{K B} \approx \gamma}$ & $\checkmark$ \\
\hline $\mathrm{CM}$ & $\frac{\mathcal{K B} \approx \alpha \quad \mathcal{K B} \approx \gamma}{\mathcal{K} \mathcal{B} \cup\{\alpha\} \approx \gamma}$ & $\checkmark$ \\
\hline LLE & $\frac{\mathcal{K B} \cup\{\alpha\} \approx \gamma \quad \models \alpha \equiv \beta}{\mathcal{K B} \cup\{\beta\} \approx \gamma}$ & $\checkmark$ \\
\hline RW & $\frac{\mathcal{K} \mathcal{B} \approx \alpha \quad \alpha \models \gamma}{\mathcal{K} \mathcal{B} \approx \gamma}$ & $\checkmark$ \\
\hline OR & $\frac{\mathcal{K B} \cup\{\alpha\} \approx \gamma \quad \mathcal{K B} \cup\{\beta\} \approx \gamma}{\mathcal{K B} \cup\{\alpha \vee \beta\} \approx \gamma}$ & under extra axioms \\
\hline $\mathrm{RM}$ & $\frac{\mathcal{K B} \approx \gamma \quad \mathcal{K B} \not \approx \neg \alpha}{\mathcal{K B} \cup\{\alpha\} \approx \gamma}$ & under extra axioms \\
\hline
\end{tabular}

$\mathcal{K B}$ is a canonical $\mathcal{D} \mathcal{L}^{\mathrm{N}}$ knowledge base;

$\alpha$ and $\beta$ range over $\mathcal{D} \mathcal{L}$ assertions and (strong) concept and role inclusions;

$\gamma$ ranges over $\mathcal{D} \mathcal{L}^{\mathrm{N}}$ assertions and $\mathcal{D} \mathcal{L}^{\mathrm{N}}$ concept/role inclusions;

nonstandard DL axioms $\alpha \vee \beta, \neg \beta$ can be simulated, e.g. with the universal role;

$\approx$ denotes the nonmonotonic consequence relation of $\mathcal{D} \mathcal{L}^{\mathrm{N}}$ and $\models$ classical inference.

Although these postulates are not necessarily desiderata, due to the loose correspondence between their motivations and $\mathcal{D} \mathcal{L}^{\mathrm{N}}$ 's goals and semantics (cf. [715]), we regard them as a useful technical tool for comparison, since the validity of the postulates has been extensively investigated in most nonmonotonic logics.

There exist several versions of the postulates; all of them contain postulates that are incompatible with $\mathcal{D} \mathcal{L}^{\mathrm{N}}$ 's novel way of highlighting unresolved conflicts through inconsistent prototypes, for debugging purposes. So, hereafter, we assume that all unresolved conflicts have been fixed, as recommended by this knowledge engineering methodology.

The first version of the postulates - illustrated in Table 2 - is the verbatim instantiation of the original, meta-level postulates. A consequence relation that satisfies the KLM postulates is called rational. It is called preferential if it satisfies all rules but RM, and cumulative if it satisfies all rules but RM and OR. With respect to this version of the postulates, $\mathcal{D} \mathcal{L}^{\mathrm{N}}$ 's consequence relation $(\approx)$ is cumulative 6

\footnotetext{
${ }^{6}$ Obviously, REF and RW always hold, because $\mathcal{D} \mathcal{L}^{\mathrm{N}}$ is closed under classical inference. Moreover rules CT, CM, and LLE are sound by [15, Thm 1].
} 
It is interesting to note that the rational closure of DLs itself is not rational w.r.t. this version of the postulates, e.g. it fails to satisfy the OR rule 15. On the contrary, $\mathcal{D} \mathcal{L}^{\mathrm{N}}$ can be made fully rational by making it a little more similar to typicality logic and rational closure, in the following respect. The semantic of typicality and rational closure forces each consistent concept to have a normal instance, through the smoothness property of preferential models and the notion of canonical model 29. A similar condition can be enforced in $\mathcal{D} \mathcal{L}^{\mathrm{N}}$ through the axioms $\neg(\mathrm{N} C \sqsubseteq \perp)$, for all consistent $C$ occurring in $\mathcal{K} \mathcal{B}$ or in the query. The knowledge bases so extended satisfy all the postulates in Table Q 2 . The above results provide an immediate comparison with the consequence relations of Circumscribed DLs (that are preferential) and those of Default and Autoepistemic DLs (that are not cumulative).

\begin{tabular}{|c|c|c|}
\hline Name & Rule schema & Sound \\
\hline $\mathrm{REF}_{n}$ & $\overline{C \sqsubseteq_{n} C}$ & $\checkmark$ \\
\hline $\mathrm{CT}_{n}$ & $\frac{C \sqsubseteq_{n} D \quad C \sqcap D \sqsubseteq_{n} E}{C \sqsubseteq_{n} E}$ & \\
\hline $\mathrm{CM}_{n}$ & $\frac{C \sqsubseteq_{n} D \quad C \sqsubseteq_{n} E}{C \sqcap D \sqsubseteq_{n} E}$ & \\
\hline $\mathrm{LLE}_{n}$ & $\frac{C \sqsubseteq_{n} E \quad \mathcal{S} \models C \equiv D}{D \sqsubseteq_{n} E}$ & \\
\hline $\mathrm{RW}_{n}$ & $\frac{C \sqsubseteq_{n} D \quad \mathcal{S} \models D \sqsubseteq E}{C \sqsubseteq_{n} E}$ & \\
\hline $\mathrm{OR}_{n}$ & $\frac{C \sqsubseteq_{n} E \quad D \sqsubseteq_{n} E}{C \sqcup D \sqsubseteq_{n} E}$ & \\
\hline $\mathrm{RM}_{n}$ & $\frac{C \sqsubseteq_{n} E \quad C \nsubseteq_{n} \neg D}{C \sqcap D \sqsubseteq_{n} E}$ & \\
\hline
\end{tabular}

$\mathcal{S}$ is the strong part of a knowledge base

Table 3. Analogues of the KLM postulates for DIs 7 .

\begin{tabular}{|c|c|c|}
\hline Name & Rule schema & Sound \\
\hline $\mathrm{REF}^{N}$ & \multicolumn{2}{|l|}{$\overline{\mathrm{NC} \sqsubseteq C}$} \\
\hline $\mathrm{CT}^{N}$ & $\frac{\mathrm{N} C \sqsubseteq D \quad \mathrm{~N}(C \sqcap D) \sqsubseteq E}{\mathrm{~N} C \sqsubseteq E}$ & partly \\
\hline $\mathrm{CM}^{N}$ & $\frac{\mathrm{N} C \sqsubseteq D \quad \mathrm{~N} C \sqsubseteq E}{\mathrm{~N}(C \sqcap D) \sqsubseteq E}$ & \multirow[t]{2}{*}{ partly } \\
\hline $\operatorname{LLE}^{N}$ & $\frac{\mathrm{N} C \sqsubseteq E \quad C \equiv D}{\mathrm{~N} D \sqsubseteq E}$ & \\
\hline $\mathrm{RW}^{N}$ & $\frac{\mathrm{N} C \sqsubseteq D \quad D \sqsubseteq E}{\mathrm{~N} C \sqsubseteq E}$ & $\checkmark$ \\
\hline $\mathrm{OR}^{N}$ & $\frac{\mathrm{N} C \sqsubseteq E \quad \mathrm{~N} D \sqsubseteq E}{\mathrm{~N}(C \sqcup D) \sqsubseteq E}$ & \multirow[b]{2}{*}{ partly } \\
\hline $\mathrm{RM}^{N}$ & $\frac{\mathrm{N} C \sqsubseteq E \quad \mathrm{~N} C \nsubseteq \neg D}{\mathrm{~N}(C \sqcap D) \sqsubseteq E}$ & \\
\hline , & $E$ range over $\mathcal{D} \mathcal{L}$ cor & epts \\
\hline
\end{tabular}

Table 4. $\mathcal{D} \mathcal{L}^{\mathrm{N}}$ Candidate inference rules inspired by KLM postulates

Several logics internalize the nonmonotonic consequence relation and push the KLM postulates to the object level (e.g. [20|21|22|18|16|28|29]). The resulting postulates for $\mathcal{D} \mathcal{L}^{\mathrm{N}}$ are reported in Table 3 Their validity is clearly affected by overriding. For instance, if the second premise of $\mathrm{CM}_{n}$ were overridden, then there would be no logical ground for supporting the conclusion. However, if the premises are not overridden, then all the postulates of Table 3 are valid in $\mathcal{D} \mathcal{L}^{\mathrm{N}}$. It is interesting to note that a similar phenomenon can be observed in Lehmanns account of default reasoning 34 . In Sec. 6 Lehmann exhibits a knowledge base 
with no (consistent) rational closure; however it has a lexicographic closure because the latter ignores all overridden defaults. The second interesting remark is that two of these postulates unconditionally hold in most practically interesting cases: (i) the $\mathrm{OR}_{n}$ rule holds if the priority relation is specificity; (ii) $\mathrm{LLE}_{n}$ holds whenever the priority relation is not sensitive to syntactic details i.e. treats logically equivalent DIs in the same way (like specificity does).

Another internalized version of the postulates, analogous to those satisfied by typicality logics 28129] is reported in Table 4 It can be shown that typicality DLs satisfy these postulates only because the normality criterion is assumed to be concept-independent (i.e. if John is more typical than Mary as a driver, then he must also be more typical than Mary as a worker, as a tax payer, and so on) [15. $\mathcal{D} \mathcal{L}^{\mathrm{N}}$ does not adopt this strong assumption: in $\mathcal{D} \mathcal{L}^{\mathrm{N}}$ each concept may have its own notion of what is more normal or standard and - for this reason it does not universally satisfy $\mathrm{CT}^{N}$ and $\mathrm{CM}^{N}$. So an interesting open question is whether the postulates of Table 4 can possibly be satisfied by a logic that does not rely on a unique, concept-independent normality relation.

Again, $\mathcal{D} \mathcal{L}^{\mathrm{N}}$ can be made fully rational (with respect to this version of the postulates) by making it more similar to rational closure. Consider the restriction of $\mathcal{D} \mathcal{L}^{\mathrm{N}}$ where $\mathrm{N}$ does not explicitly occur in $\mathrm{KB}$ ( $\mathrm{N}$ can be used only in queries). In practice, this means that role fillers cannot be forced to be normal, similarly to what inevitably happens in rational closure and default DLs, due to the limitations of these logics. Under this restriction, all postulates in Table 4 hold [15. Thm 4, 5,6].

\section{Conclusions and future work}

$\mathcal{D} \mathcal{L}^{\mathrm{N}}$ addresses a number of drawbacks that affect the nonmonotonic semantics of DLs. It has been designed with a particular attention to practical issues that hinder the adoption of nonmonotonic semantics in OWL2 and its profiles, including expressiveness limitations, and complexity problems. Moreover, $\mathcal{D} \mathcal{L}^{\mathrm{N}}$ reasoning can be easily reduced to classical reasoning, thereby leveraging the high-quality, well-engineered implementations of DL reasoning. In the light of these properties, $\mathcal{D} \mathcal{L}^{\mathrm{N}}$ compares favorably to the other nonmonotonic DLs, as summarized in Table 1 .

Interestingly, even if KLM postulates played no role in $\mathcal{D} \mathcal{L}^{\mathrm{N}}$, s design, $\mathcal{D} \mathcal{L}^{\mathrm{N}}$ satisfies to a large extent the major meta-level and internalized versions of the postulates. The postulates in Table 3 hold up to overriding, as in Lehmann's lexicographic closure. Moreover, $\mathcal{D} \mathcal{L}^{\mathrm{N}}$ is flexible enough to satisfy all the other postulates by means of additional axioms or syntactic restrictions that make $\mathcal{D} \mathcal{L}^{\mathrm{N}}$ more similar to typicality logics and rational closure.

Many different directions deserve further investigation. From a semantic viewpoint, we mentioned in Section 4.2 that the KLM postulates OR and RM are sound in $\mathcal{D} \mathcal{L}^{\mathrm{N}}$ under the assumption that consistent concepts have at least one typical individual. Taking inspiration from typicality logics, variants of $\mathcal{D} \mathcal{L}^{\mathrm{N}}$ can be investigated where this assumption is hard-coded in the semantics. This 
should be done with some care, though: if too many individuals were forced to be normal, then the undesirable closed-world assumption effects described in 77, that affect typicality logics, might be introduced in $\mathcal{D} \mathcal{L}^{\mathrm{N}}$.

The study of the logical properties of $\mathcal{D} \mathcal{L}^{\mathrm{N}}$ can be refined by investigating the mutual relationships between DIs and their effects on normality concepts. In particular, it would be interesting to investigate hybrid versions of the postulates, whose premises are taken from Table 3 while consequents are taken from Table4.

Finally, one of the primary strengths of $\mathcal{D} \mathcal{L}^{\mathrm{N}}$ is that it preserves the tractability of the low-complexity DLs underlying the OWL2-EL and OWL2-QL profiles. However, asymptotic tractability alone does not suffice for practical purposes. In [13, two optimization techniques have been successfully applied to obtain realtime query answering over large knowledge bases. One optimization is based precisely on a suitably modified module extraction algorithm, that so far constitutes the most effective optimization technique for $\mathcal{D} \mathcal{L}^{\mathrm{N}}$ (excluding combined approaches). The other optimization, called optimistic method, reduces the number of retractions (an expensive class of operations in incremental reasoning). $\mathcal{D} \mathcal{L}^{\mathrm{N}}$ 's module extractor, however, proved to be less effective for KBs that contain many explicit occurrences of the normality concepts, and for those with nonempty ABoxes (due to the lesser effectiveness of the underlying classical module extractors in such contexts). To overcome these problematic cases, we plan to improve the module extractor for $\mathcal{D} \mathcal{L}^{\mathrm{N}}$ by discarding the normality concepts (and related axioms) and assertions that are irrelevant to a given query.

\section{References}

1. A. Artale, D. Calvanese, R. Kontchakov, and M. Zakharyaschev. The DL-lite family and relations. J. Artif. Intell. Res. (JAIR), 36:1-69, 2009.

2. F. Baader, S. Brandt, and C. Lutz. Pushing the EL envelope. In Proc. of the $19^{\text {th }}$ Int. Joint Conf. on Artificial Intelligence, IJCAI-05, pages 364-369. Professional Book Center, 2005.

3. F. Baader and B. Hollunder. Embedding defaults into terminological knowledge representation formalisms. J. Autom. Reasoning, 14(1):149-180, 1995.

4. F. Baader and B. Hollunder. Priorities on defaults with prerequisites, and their application in treating specificity in terminological default logic. J. Autom. Reasoning, 15(1):41-68, 1995.

5. F. Baader, D. L. McGuiness, D. Nardi, and P. Patel-Schneider. The Description Logic Handbook: Theory, implementation and applications. Cambridge University Press, 2003.

6. P. A. Bonatti. Rational closure for all description logics. Artif. Intell., 274:197-223, 2019.

7. P. A. Bonatti, M. Faella, I. Petrova, and L. Sauro. A new semantics for overriding in description logics. Artif. Intell., 222:1-48, 2015.

8. P. A. Bonatti, M. Faella, and L. Sauro. Defeasible inclusions in low-complexity DLs: Preliminary notes. In Proceedings of the 21st International Joint Conference on Artificial Intelligence (IJCAI'09), Pasadena, California, USA, 2009.

9. P. A. Bonatti, M. Faella, and L. Sauro. EL with default attributes and overriding. In Int. Semantic Web Conf. (ISWC 2010), volume 6496 of LNCS, pages 64-79. Springer, 2010. 
10. P. A. Bonatti, M. Faella, and L. Sauro. Adding default attributes to EL++. In W. Burgard and D. Roth, editors, AAAI. AAAI Press, 2011.

11. P. A. Bonatti, M. Faella, and L. Sauro. Defeasible inclusions in low-complexity DLs. J. Artif. Intell. Res. (JAIR), 42:719-764, 2011.

12. P. A. Bonatti, C. Lutz, and F. Wolter. The complexity of circumscription in DLs. J. Artif. Intell. Res. (JAIR), 35:717-773, 2009.

13. P. A. Bonatti, I. M. Petrova, and L. Sauro. Optimizing the computation of overriding. In The Semantic Web - ISWC 2015 - 14th International Semantic Web Conference, Bethlehem, PA, USA, October 11-15, 2015, Proceedings, Part I, pages 356-372, 2015.

14. P. A. Bonatti and P. Samarati. Logics for authorization and security. In Logics for Emerging Applications of Databases, pages 277-323. Springer, 2003.

15. P. A. Bonatti and L. Sauro. On the logical properties of the nonmonotonic description logic $\mathrm{dl}^{\mathrm{n}}$. Artif. Intell., 248:85-111, 2017.

16. K. Britz, T. Meyer, and I. J. Varzinczak. Semantic foundation for preferential description logics. In D. Wang and M. Reynolds, editors, AI 2011: Advances in Artificial Intelligence - 24th Australasian Joint Conference, Perth, Australia, December 5-8, 2011. Proceedings, volume 7106 of Lecture Notes in Computer Science, pages 491-500. Springer, 2011.

17. D. Calvanese, G. De Giacomo, D. Lembo, M. Lenzerini, and R. Rosati. DL-Lite: Tractable description logics for ontologies. In Proc. of AAAI 2005, pages 602-607, 2005.

18. G. Casini, T. Meyer, K. Moodley, and R. Nortje. Relevant closure: A new form of defeasible reasoning for description logics. In E. Fermé and J. Leite, editors, Logics in Artificial Intelligence - 14th European Conference, JELIA 2014, Funchal, Madeira, Portugal, September 24-26, 2014. Proceedings, volume 8761 of Lecture Notes in Computer Science, pages 92-106. Springer, 2014.

19. G. Casini, T. Meyer, K. Moodley, and I. J. Varzinczak. Towards practical defeasible reasoning for description logics. In T. Eiter, B. Glimm, Y. Kazakov, and M. Krötzsch, editors, Description Logics, volume 1014 of CEUR Workshop Proceedings, pages 587-599. CEUR-WS.org, 2013.

20. G. Casini and U. Straccia. Rational closure for defeasible description logics. In T. Janhunen and I. Niemelä, editors, JELIA, volume 6341 of Lecture Notes in Computer Science, pages 77-90. Springer, 2010.

21. G. Casini and U. Straccia. Defeasible inheritance-based description logics. In T. Walsh, editor, IJCAI 2011, Proceedings of the 22nd International Joint Conference on Artificial Intelligence, Barcelona, Catalonia, Spain, July 16-22, 2011, pages 813-818. IJCAI/AAAI, 2011.

22. G. Casini and U. Straccia. Defeasible inheritance-based description logics. J. Artif. Intell. Res. (JAIR), 48:415-473, 2013.

23. G. Casini, U. Straccia, and T. Meyer. A polynomial time subsumption algorithm for nominal safe elo ${ }^{\perp}$ under rational closure. Inf. Sci., 501:588-620, 2019.

24. F. M. Donini, D. Nardi, and R. Rosati. Autoepistemic description logics. In IJCAI (1), pages 136-141, 1997.

25. F. M. Donini, D. Nardi, and R. Rosati. Description logics of minimal knowledge and negation as failure. ACM Trans. Comput. Log., 3(2):177-225, 2002.

26. L. Giordano, V. Gliozzi, N. Olivetti, and G. Pozzato. Reasoning about typicality in preferential description logics. In Proc. of Logics in Artificial Intelligence, 11th European Conference, JELIA 2008, volume 5293 of Lecture Notes in Computer Science. Springer, 2008. 
27. L. Giordano, V. Gliozzi, N. Olivetti, and G. L. Pozzato. Prototypical reasoning with low complexity description logics: Preliminary results. In E. Erdem, F. Lin, and T. Schaub, editors, LPNMR, volume 5753 of Lecture Notes in Computer Science, pages 430-436. Springer, 2009.

28. L. Giordano, V. Gliozzi, N. Olivetti, and G. L. Pozzato. A non-monotonic description logic for reasoning about typicality. Artif. Intell., 195:165-202, 2013.

29. L. Giordano, V. Gliozzi, N. Olivetti, and G. L. Pozzato. Semantic characterization of rational closure: From propositional logic to description logics. Artif. Intell., 226:1-33, 2015.

30. L. Giordano, V. Gliozzi, G. L. Pozzato, and R. Renzulli. An efficient reasoner for description logics of typicality and rational closure. In Proceedings of the 30th International Workshop on Description Logics, Montpellier, France, July 18-21, 2017, 2017.

31. L. Giordano, N. Olivetti, V. Gliozzi, and G. L. Pozzato. ALC + T: a preferential extension of description logics. Fundam. Inform., 96(3):341-372, 2009.

32. Y. Kazakov. Riq and sroiq are harder than shoiq. In G. Brewka and J. Lang, editors, $K R$, pages 274-284. AAAI Press, 2008.

33. S. Kraus, D. J. Lehmann, and M. Magidor. Nonmonotonic reasoning, preferential models and cumulative logics. Artif. Intell., 44(1-2):167-207, 1990.

34. D. Lehmann. Another perspective on default reasoning. Ann. Math. Artif. Intell., 15(1):61-82, 1995.

35. D. J. Lehmann and M. Magidor. What does a conditional knowledge base entail? Artif. Intell., 55(1):1-60, 1992.

36. T. Lukasiewicz. Expressive probabilistic description logics. Artif. Intell., 172(67):852-883, 2008.

37. J. McCarthy. Applications of circumscription to formalizing common sense knowledge. Artificial Intelligence, 28:89-116, 1986.

38. M. Pensel. A Lightweight Defeasible Description Logic in Depth. PhD thesis, TU Dresden, 2019.

39. A. L. Rector. Defaults, context, and knowledge: Alternatives for OWL-indexed knowledge bases. In Pacific Symposium on Biocomputing, pages 226-237. World Scientific, 2004

40. E. Sandewall. Defeasible inheritance with doubt index and its axiomatic characterization. Artif. Intell., 174(18):1431?1459, Dec. 2010.

41. R. Stevens, M. E. Aranguren, K. Wolstencroft, U. Sattler, N. Drummond, M. Horridge, and A. L. Rector. Using OWL to model biological knowledge. International Journal of Man-Machine Studies, 65(7):583-594, 2007. 